\title{
Sensibilité de trois espèces de Chironomus (Diptera) à huit insecticides utilisés en démoustication
}

\author{
G. Sinegre 1 \\ M. Babinot ${ }^{1}$ \\ G. Vigo 1 \\ J.N. Tourenq ${ }^{2}$
}

Mots clés : Chironomides, insecticides, inhibiteurs de mue, toxines bactériennes.

Des expérimentations de terrain en parcelles limitées ont permis, à partir de piégeages d'adultes, d'évaluer l'impact de huit insecticides sur des populations larvaires composées de $82 \%$ de Chironomus salinarius, de $12 \%$ de Chironomus halophilus et de Chironomus plumosus et $6 \%$ d'autres espèces non identifées. Le diflubenzuron et les toxines de Bacillus thuringiensis $\mathrm{H} 14$ et Bacillus sphaericus n'ont pas d'effet marqué aux doses détruisant les larves de moustiques. A ces mêmes doses, le fénitrothion, le téméphos et la deltaméthrine limitent fortement l'émergence. Le chlorpyrifos et le $\mathrm{S}$ 31183 (juvénoïde Sumitomo) sont très toxiques.

Sensitiveness of three Chironomus (Diptera) species to eight larvicides used for mosquito control.

Keywords : Chironomidae, insecticides, insect growth regulator, bacterial toxins.

The impact of eight insecticides on larval populations of Chironomids was evaluated by adult emergence trapping in field experiments using limited plots. The populations were composed of $82 \%$ of Chironomus salinarius, $12 \%$ of Chironomus halophilus and Chironomus plumosus and $6 \%$ of unidentified species. Diflubenzuron and the toxins of Bacillus thuringiensis $\mathrm{H} 14$ and Bacillus sphaericus have no noticeable effect at doses killing mosquito larvac. At the same doses, fenitrothion, temephos, and deltamethrin heavily inhibited adult emergence. Chlorpyrifos and S 31183(a juvenoid of Sumitomo) were highly toxic.

\section{Introduction}

Les adultes de Chironomides, diptères non piqueurs, sont fréquemment confondus avec les moustiques par toute personne non avertie. Leur prolifération, à certaines périodes de l'année, cause une gène réelle à l'économie et au tourisme, notamment sur le littoral Méditerranéen français. Leur densité est telle sur ce littoral que les tours de contrôle de certains aéroports ont parfois du mal à assumer leur fonction et que certains hypermarchés ou commerces divers ont été contraints à ưne

\footnotetext{
1. Démoustication Méditerranée, B.P. 6036, F. 34030 Montpellier Cedex 01.

2.Laboratoire d'Hydrobiologie, Université Paul-Sabatier, UA 695 du CNRS, 118, route de Narbonne, F. 31062 Toulouse Cédex.
}

fermeture momentanée. Plusieurs travaux rapportent cette nuisance et au-delà, les risques et manifestations allergiques encourus tant en France qu'à l'étranger (Jolivet 1974, Tabaru 1975, Ali \& Mulla 1978, Ali 1980, Ceretti et al. 1985). En zone naturelle, on note un équilibre harmonieux entre diverses espèces de chironomides et autres représentants de la faune des milieux marécageux saumâtres. Dans les zones à forte densité de population, l'origine du déséquilibre semble en partie lié à la pollution organique des étangs littoraux jouxtant ces zones.

En terme de biomasse, les Chironomides, avec plus de 80 espèces recensées dans le Midi de la France (Tourenq 1975, 1976), sont un utile maillon des chaînes alimentaires, tant pour la faune aquatique que semi aquatique ou terrestre. Ils contribuent par ailleurs largement au processus épuratoire des eaux. 
Le Service de Démoustication du Littoral Languedoc-Roussillon (E.I.D.), saisi par les autorités locales pour engager la lutte, se trouve ainsi confronté au double problème de la sauvegarde et de la destruction. Un programme d'études en cours doit permettre de délimiter les zones où l'intervention s'impose et de définir les méthodes et moyens les mieux adaptés. Dans ce cadre, une expérimentation de terrain a été réalisée pour évaluer l'impact de quelques produits de synthèse sur les populations larvaires des espèces les plus fréquemment impliquées dans la nuisance. Huit insecticides d'appartenances chimiques diverses ont été retenus. Ils sont d'usage courant en lutte anti moustique. La plupart d'entre-eux ont une activité reconnue sur certaines espèces de Chironomides en Californie (Ali \& Mulla 1979), Italie (Ali \& Majori 1984) et Japon (Tabaru 1985).

\section{Matériel et méthode}

Des cadres en plastique transparent et biologiquement inertes de $0,25 \mathrm{~m}^{2}$ de surface et $50 \mathrm{~cm}$ de hauteur sont enfoncés sur $5 \mathrm{~cm}$ dans le substrat vaseux d'un marais temporairement inondé sur 30 à $40 \mathrm{~cm}$ et n'ayant, en principe, jamais reçu d'insecticides. Chaque cadre, recouvert d'un tulle moustiquaire, permet le piégeage des imagos nouvellement éclos. L'application des insecticides est réalisée dans la semaine qui suit l'apparition des premières émergences, généralement début mai. Huit cadres servent de témoin à raison d'un par insecticide. Trois doses par insecticides exprimées en gramme/ha de produit actif pour les composés de synthèse et en litre/ha pour les formulations bactériennes, ont été utilisées : une dose médiane égale à celle généralement efficace sur larves de moustiques, une dose trois fois moindre et une dose trois fois plus élevée.

- A partir du premier jour de traitement et durant un mois environ, les adultes émergents ont été dénombrés deux fois par semaine. Les déterminations ont reposé sur les critères taxonomiques des imagos mâles piégés dans les cadres témoins. Chironomus salinarius représent ait $82 \%$ des individus, Chironomus halophilus et Chironomus plumosus $12 \%$, le reliquat $(6 \%)$ étant composé de plusieurs espèces non identifiées.

- Les insecticides ont été appliqués après mise en suspension extamporanée d'une quantité adéquate de formulation commerciale dans $250 \mathrm{ml}$ d'eau. Il s'agit de trois organophosphorés : téméphos, chlorpyrifos et fénitrothion ; deux régulateurs de croissance : diflubenzuron et S 31193 ou 2 - (1 méthyl 1 - 2 - (4 phenoxyphenox) ethoxy pyridine) ; deux agents bactériens: Bacillus thuringiensis sérotype H 14 et Bacillus sphaericus souche 2362 .

\section{Résultat et discussion}

Les résultats, présentés sur les figures 1 à 3 , appellent les commentaires suivants :

- Parcelles témoin : d'une manjère générale, on observe une bonne similitude quant aux éclosions d'adultes en fonction du temps. Celles-ci présentent un premier pic pendant la première quinzaine suivi d'un deuxième pic plus important la troisième semaine.

- Téméphos : cet organo-phosphoré est couramment utilisé par l'E.1.D. depuis vingt ans en Languedoc-Roussillon pour lutter contre les larves de moustiques. Une dose de $50 \mathrm{~g} / \mathrm{ha}$ tend à réduire sensiblement les émergences, sans les enrayer totalement. Au total, 2.280 imagos de Chironomides ont éclos dans la parcelle traitée, contre 3.400 en parcelle témoin. La réduction d'effectif est donc voisine de $33 \%$ seulement. A la dose de $150 \mathrm{~g} / \mathrm{ha}$, classiquement utilisée lors des traitements de démoustication, les éclosions apparaissent seulement au cours des sept premiers jours qui suivent le traitement, ce qui tend à montrer que les larves au stade IV des espèces de Chironomides peuvent survivre à cette dose, contrairement aux larves les plus jeunes. Sur la base des effectifs piégés, la réduction par rapport au témoin atteint $75 \%$. Avec des doses de 450 $\mathrm{g} / \mathrm{ha}$, la quasi totalité des larves est détruite, les quelques rares individus éclos la première semaine après traitement peuvent provenir des nymphes ou des larves prêtes à se nymphoser présentes au moment de l'épandage et peu sensibles à cet insecticide.

- Chlorpyrifos : une dose de $15 \mathrm{~g} / \mathrm{ha}$ est déjà très toxique pour les larves des trois Chironomus. On a dénombré respectivement 22 et 33 adultes seulement lors des contrôles effectués 4 et 7 jours après traitement contre 502 à 508 en parcelle témoin. Aucune éclosion ne s'est produite après la première semaine. Cet insecticide organophosphoré est connu pour son large spectre d'activité mais, à ce jour, il n'est utilisé par les Services de démoustication que dans le cadre de la lutte urbaine en eaux polluées. 

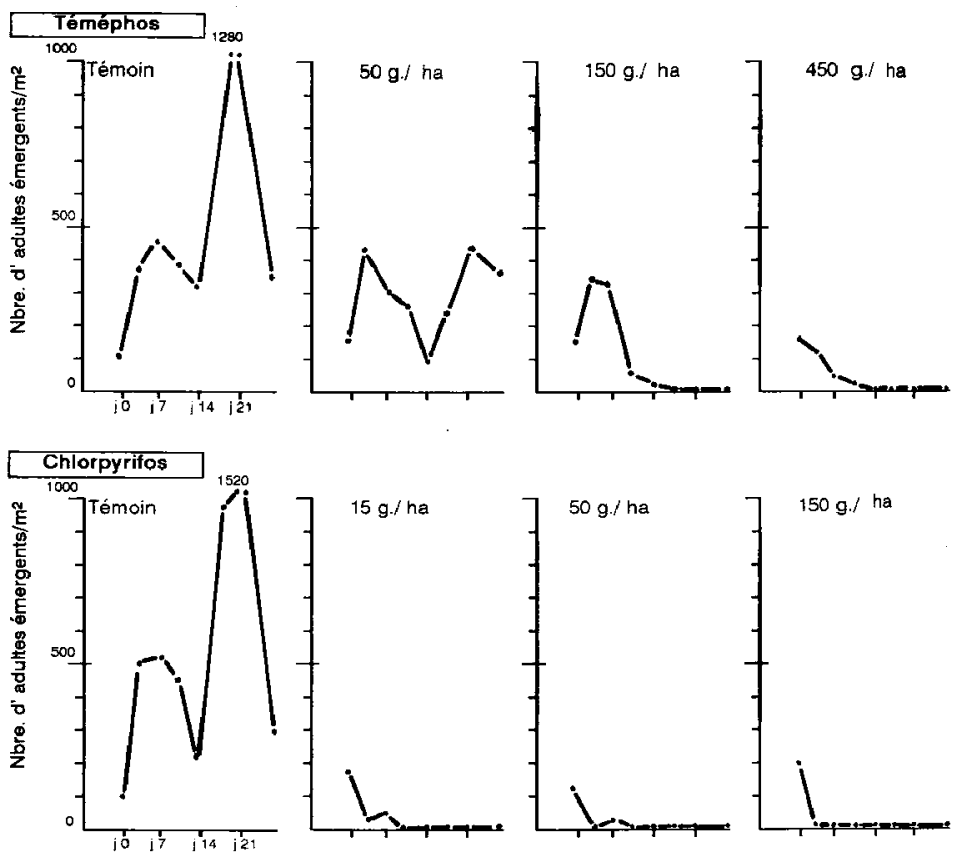

Fénitrothion
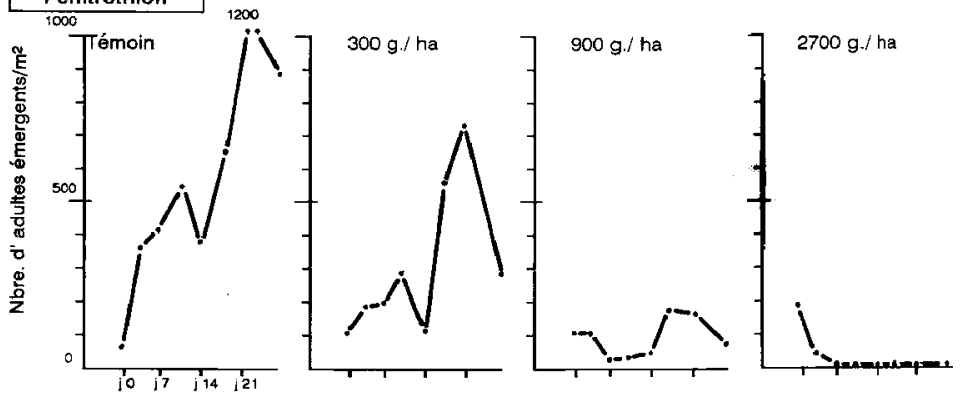

Fig. 1. Courbes d'émergences post-traitements d'adultes de Chironomides en gîtes traités et témoin. 

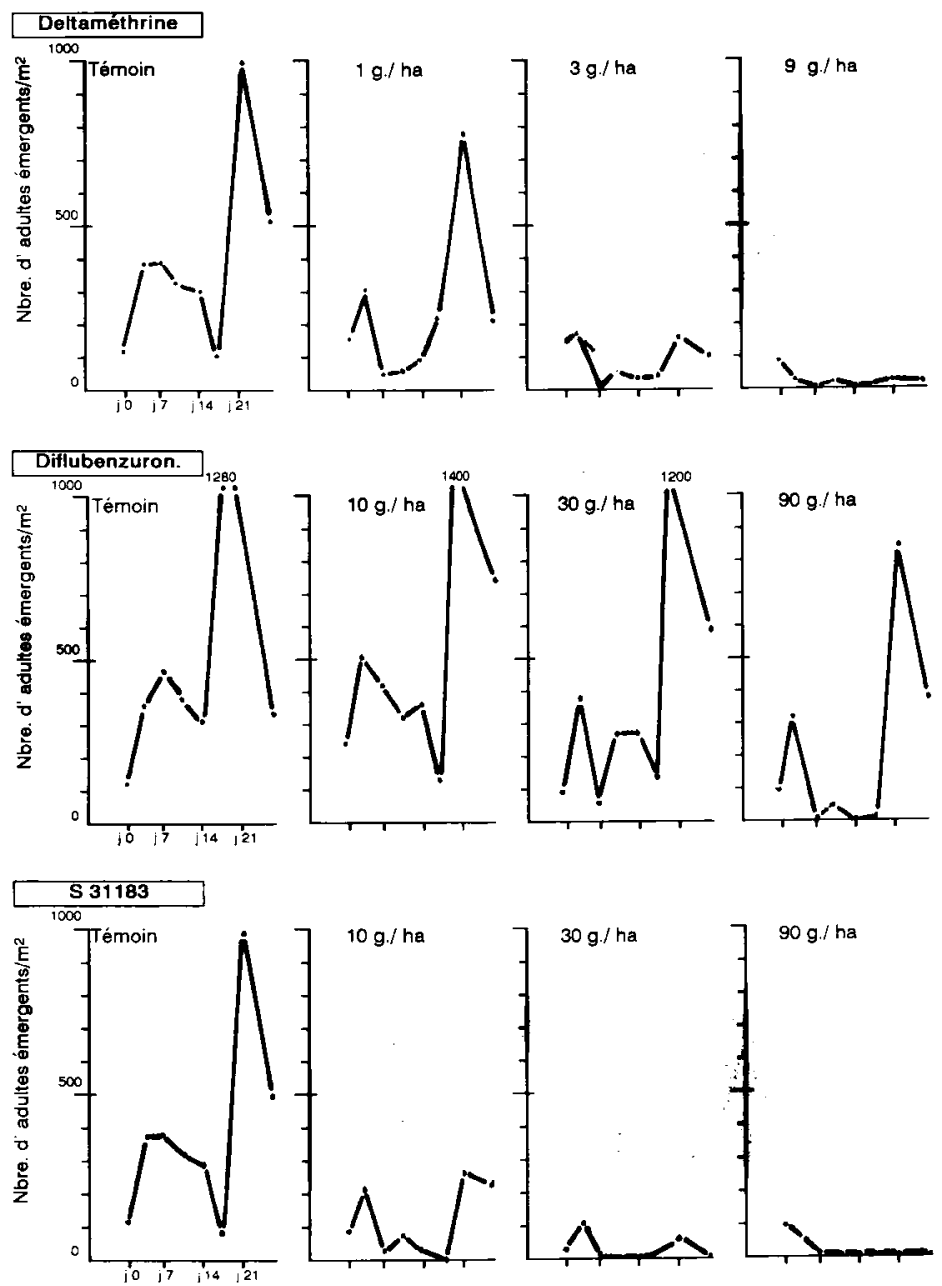

Fig. 2. Courbes d'émergences post-traitements d'adultes de Chironomides en gites traités et témoin. 

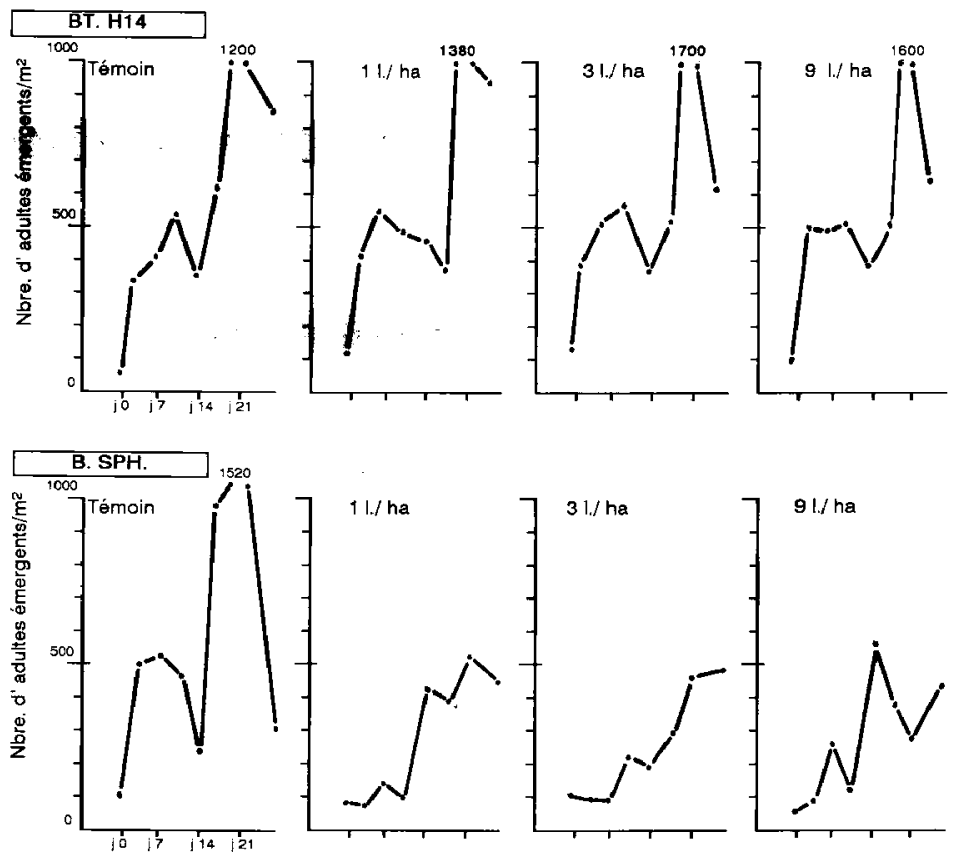

Fig. 3. Courbes d'émergences post-traitements d'adultes de Chironomides en gîtes traités et témoin.

- Fénitrothion : c'est le larvicide le plus couramment utilisé de l'automne au printemps pour dêtruire les larves de moustiques dans les zones marécageuses du Midi de Ia France. La dose généralement appliquée est de $600 \mathrm{~g} / \mathrm{ha}$ mais elle peut atteindre $900 \mathrm{~g} / \mathrm{ha}$ dans certains cas. A cette dose, les éclosions de Chironomides sont réduites de $86 \%$ par rapport au témoin. A $300 \mathrm{~g} / \mathrm{ha}$, l'effectif semble seulement réduit de moitié.

- Deltaméthrine: le très large spectre d'action de ce pyréthrinoïde a conduit l'E.I.D. à réserver son emploi aux seuls traitements sporadiques contre les adultes de moustiques. Utilisé dans ce cadre à $\mathrm{l} g$ /ha, on note un impact modéré sur les larves de Chironomides avec réduction d'émergence de $47 \%$ par rapport aux témoins. Toutefois, il est évident que la micronisation très fine, pratiquée dans ce genre d'intervention détruit tout autant les adultes de Chironomides que ceux de moustiques dans les mêmes gîtes de repos. A $3 \mathrm{~g} / \mathrm{ha}$, dose nécessaire pour tuer les larves de moustique, on note un impact majeur sur les larves de Chironomides, avec réduction d'effectif de $83 \%$.

- Diflubenzuron : cet insecticide benzoyl-urée, agent potentiel de lutte contre les larves de moustiques, n'est pas encore utilisé dans le Sud de la France. Si tel était le cas, il serait nécessaire d'appliquer une dose d'au moins $30 \mathrm{~g} / \mathrm{ha}$. Avec une 
réduction d'effectif de $20 \%$ seulement cette dose agit modérément sur les larves de Chironomides, ce que confirme la dose inopérante de $10 \mathrm{~g} / \mathrm{ha}$, puisque le nombre total d'adultes émergé est supérieur au nombre de la parcelle témoin. A $90 \mathrm{~g} / \mathrm{ha}$, la réduction d'effectif est de près de la moitié $(48 \%)$. Les traitements contre les larves de moustiques réalisés avec ce produit seraient certainement sans effet marquant sur les populations larvaires de Chironomides.

- $S 31183$ : cette molécule expérimentale de type ecdysoïde est très toxique pour les moustiques au moment de leur mue larvo-nymphale ou nymphoimaginale. On note également une toxicité très élevée sur les Chironomides dont l'effectif émergent est respectivement réduit de $75 \%, 95 \%$ et $97 \%$ aux doses de 10,30 et $90 \mathrm{~g} / \mathrm{ha}$.

- Bacillus thuringiensis $H$ 14: les émergences d'adulte en parcelles traitées sont quantitativement identiques aux émergences témoin quelle que soit la dose/ha utilisée (entre 1 et 9 de Vectolex ${ }^{(\text {is) }}$ ). En milieu naturel, cette spécialité est efficace sur moustiques à $2 \mathrm{l}$ /ha environ mais, à ce jour, son emploi est réservé aux seuls traitements de zones protégées. $\mathrm{Au}$ vu de ces résultats, on notera que les traitements de démoustication dans ces zones seraient sans effet toxique sur les larves de Chironomides, tout au moins sur les trois espèces de Chironomus considérées dans cette étude.

- Bacillus sphaericus : les courbes se rapportant aux éclosions en parcelles traitées tendent à montrer une réduction d'effectif par rapport au témoin. Toutefois, il n'apparaît pas de corrélation entre les différentes doses de $B$. sphaericus et le nombre d'adultes émergents. Aussi, l'on peut s'interroger sur l'origine de la différence entre les parcelles traitées et la parcelle témoin. De nombreux travaux ont montré que la toxine de $\boldsymbol{B}$. sphaericus est seulement active sur les larves de moustiques, et particulièrement sur celles de Culex. L'absence de corrélation semble témoigner de la non toxicité sur Chironomides. Les différences seraient alors dues aux variations de densités larvaires, parfois conséquentes, observées fréquemment d'un point à l'autre d'un même milieu. $B$. sphaericus est actuellement appliqué dans les gîtes à Culex urbains et périurbains, en particulier dans les égouts à ciel ouvert et bassins de lagunage. Ces gîtes sont souvent colonisés par Chironomus riparius. Aucune étude n'a été réalisée quant à I'impact de $B$. sphaericus sur cette espèce mais il semble très peu probable que les traitements de l'E.I.D. en réduisent le nombre.

\section{Discussion}

Il ressort de cette étude que le fénitrothion et le téméphos, aux doses utilisées en démoustication, ont un impact certain sur les larves de Chironomides testées. Toutefois, ces dernières, qui colonisent les milieux temporaires saumâtres, au même titre que les larves de moustiques, colonisent également, les milieux aquatiques permanents. En conséquence, l'impact des traitements est certainement limité dans l'espace. Il n'est, pour s'en convaincre, que de constater les nuisances évoquées précédemment, s'exprimant avec toujours plus d'acuité après 25 années de traitements anti-moustiques en LanguedocRoussillon !

La seule lutte anticulicidienne efficace qui tent à sauvegarder au mieux les Chironomides passe, d'après les résultats présentés, par l'utilisation du $B$. thuringiensis $H / 4$, encore qu'il faille respecter des doses limite d'emploi. En effet, l'étude montre que l'on dispose d'une marge de sécurité confortable vis-à-vis de $C h$. salinarius en particulier mais Garcia et al. (1980) ont noté une toxicité de $B . t H$ 14 sur certains Chironomides à $10^{6}$ spores $/ \mathrm{ml}$. De même, Ali (1981) a obtenu des réductions significatives de populations larvaires de Chironomimi et Tanytarsini à $0,5 \mathrm{mg} / 1$ de poudre de $B . t H 14$. Récemment, Pont (comm. pers.) à partir d'un travail très minutieux de terrain, réalisé dans le Midi de la France, a pu observer que la réponse au B.t H 14 est variable selon les espèces de Chironomides.

En fait, il apparaît difficile d'appréhender au mieux l'impact réel des insecticides sur les larves de Chironomides en raison de leur grande diversité spécifique en un milieu et de leurs réponses différentes aux insecticides. Ali et al. (1978) ont montré, par exemple, que le téméphos serait très toxique pour Tanytarsus s. p. et non toxique pour Procladius freemani. Ces différences se retrouvent dans notre étude : le S 31183 est hautement toxique à $30 \mathrm{~g} / \mathrm{ha}$ sur $C h$. salinarius, Ch. halophilus et Ch. plumosus alors que le diflubenzuron a peu d'effet à la même dose. La deltaméthrine et le chlorpyrifos sont également très toxiques. Tous les pyréthrinoïdes sont toxiques pour plusieurs autres espèces (Ali 1981). Le chlorpyrifos a permis de contrôler efficacement des 
populations larvaires de Chironomus sp. (Mulla \& Khasawinah, 1969), il a été reconnu plus actif que le téméphos, le fenthion ou le malathion (Ali et al. 1985).

Dans la mesure où des interventions ponctuelles seraient envisagées sur le littoral LanguedocRoussillon, l'étude montre que parmi les insecticides disponibles sur le marché, il conviendrait d'utiliser soit le chlorpyrifos soit la deltaméthrine, tous deux efficaces à faible dose. Néanmoins, compte tenu des profondeurs d'eau parfois égales ou supérieures à 1 mètre dans les gîtes permanents, riches en faune non cible, il semble bien que la lutte antilarvaire avec ces composés ne soient pas le moyen le plus sécurisant pour limiter la nuisance. Peu après l'èmergence, les Chironomides adultes forment des essaims nuptiaux extrêmement denses en bordure des étangs : des micronisations aériennes et terrestres de deltaméthrine à $1 \mathrm{~g} /$ ha seulement se sont récemment avérées efficaces et sans danger pour l'environnement. Les seules contraintes sont de travailler dans un créneau horaire très étroit et de renouveler les applications tous les deux ou trois jours jusqu'à la disparition de la nuisance. L'expérience récemment acquise en ce domaine nous montre qu'il convient, pour ce faire, de pratiquer 3 ou 4 traitements au moment des grandes éclosions dont les premières ont généralement lieu début avril.

\section{Travaux cilés.}

Ali (A.). 1980. - Nuisance chironomids and their control : a review. Bull. ent. Soc. Am., 26: 3-16.

Ali (A.). $1981 \mathrm{a}$. - Bacillus thuringiensis serovar, israelensis (ABG-6109) against chironomid and ot her nontarget aquatic invertebrates. J. Invert. Path., $38: 264-272$.

Ali (A.). 1981b. - Laboratory evaluation of organophosphate and new synthetic pyrethroid insecticide against pestiferous chironomid midges of central Florida. Mosq. News, 41 (1): $157-161$.
Ali (A.) \& Majori (G.). 1984. - A short-term investigation of Chironomid midges (Diptera : Chironomidac) problem in salt water lakes of Orbetello, Grosseto, ltaly. Mosq. News. 44 (1) : 17-21.

Ali (A.) \& Mulla (M.S.). 1978. - Chironomid population changes in an intermitent water spreading system. Mosq. News, 38 (3) 386-392.

Ali (A.) \& Mulla (M.S.). 1979. - Nuisance midge problem in southern California. Chemical control strategies. Bull. Soc. Vect. Ecol. , $4:$ 44-53.

Ali (A.), Mulla (M.S.), Pfuntner (A.R.) \& Luna (L.L.). 1978. Pestiferous midges and their control in a shallow residentialrecreational lake in southern California. Mosq. News, 38 (4) : 528-535.

Ali (A.), Majori (G.), Ceretti (G.), d'Andrea (F.) \& Ferrarese (U.). 1985. - A chironomid (Diptera, Chironomidae) midge population study and laboratory evaluation of larvicides against midges inhabiting the lagoon of Venice, Italy. $J . \mathrm{am}$. Mosq. Contr. Ass., 1 (1) : 63-68.

Ceretti (G.), Ferrarese (U.) \& Scatolin (M.). 1985. - I Chironomidi nella laguna di Venezia. Risultati delle ricerche 83-84 e proposte di lotta. Venezia, Arsenale Editrice : 59 p.

Garcia (R.), Destochers (B.) \& Tozer (W.). 1980. - - Studies on the toxicity of Bacillus thuringiensis var. israelensis against organisms found in association with mosquito larvae. Proc. ann. Conf. Calif. Mosq. Vect. Contr. Ass, 48 : 33-36.

Jolivel (P.). 1974. - Le problème des Chironomides (Diptera, Nématocère) dans le Languedoc-Roussillon (Note préliminaire). Vie et Milieu, Sér. C Biol. terr., 23 : 269-290.

Mulla (M.S.) \& Khasawinah (A.M.). 1969. - Laboratory and field evaluation of larvicides against chironomid midges. $J$. econ. Ent., 62 (1.) : 37-41.

Tabaru (Y.). 1985. - Studies on chemical control of a nuisance chironomid midge (Diptera, Chironomidae). 1. Larvicidal activity of organophosphorus insecticides against Chironomus yoshimatsui. Jap. J. santit. Zool, 36 (4) : 289-294 (en Japonais).

Tabaru (Y.). 1975. - Outbreak of chironomid midges in a polluted river and chemical control of the larvae. Jap. J. sanit. Zool., $26: 247-251$.

Tourenq (J.N.). 1975. - Recherches écologiques sur les Chironomides (Diptera) de Camargue. Thèse d'Etat, Université Paul-Sabatier, Toulouse : 424 p.

Tourenq (J. J.). 1976. - Recherches écologiques sur les Chironomides (Diptera) de Camargue. I. Etude faunistique et biogeographique. Annls Limnol., 12 (1) : 17.74. 